\title{
Ramond-Ramond field strength couplings on D-branes
}

\author{
Mohammad R. Garousi 1 \\ Department of Physics, Ferdowsi University of Mashhad \\ P.O. Box 1436, Mashhad, Iran
}

\begin{abstract}
By examining in details the disk level S-matrix element of one massless RR and one NSNS states at order $O\left(\alpha^{\prime 2}\right)$, we find the coupling of one RR and one NSNS fields on the world volume of a $\mathrm{D}_{p}$-brane. The non-zero couplings involve the first derivative of the RR field strengths $F^{(p)}, F^{(p+2)}$ and $F^{(p+4)}$. We then fix the onshell ambiguity of the couplings by requiring consistency with the linear T-duality transformations. Moreover, consistency with the non-linear T-duality requires that the RR field strength in the above couplings to be $\mathcal{F}=d \mathcal{C}$ where $\mathcal{C}=e^{B} C$.
\end{abstract}

\footnotetext{
${ }^{1}$ garousi@mail.ipm.ir
} 


\section{Introduction and results}

The dynamics of the D-branes of type II superstring theories is well-approximated by the effective world-volume field theories which consist of the sum of Dirac-Born-Infeld (DBI) and Chern-Simons (CS) actions. The DBI action describes the dynamics of the brane in the presence of the NSNS background fields. For constant background fields it can be found by requiring the consistency with nonlinear T-duality [1, 2]

$$
S_{D B I}=-T_{p} \int d^{p+1} x e^{-\phi} \sqrt{-\operatorname{det}\left(G_{a b}+B_{a b}+2 \pi \alpha^{\prime} F_{a b}\right)}
$$

where $G_{a b}$ and $B_{a b}$ are the pulled back of the bulk fields $G_{\mu \nu}$ and $B_{\mu \nu}$ onto the world-volume of D-brant 2 . The curvature corrections to this action has been found in [3] by requiring consistency of the effective action with the $O\left(\alpha^{2}\right)$ terms of the corresponding disk-level scattering amplitude [4, 5]. The on-shell ambiguity of these couplings has been removed in [6] by requiring the consistency of the couplings with linear T-duality. Moreover, this consistency fixes the couplings of non-constant dilaton and B-field at the order $O\left(\alpha^{\prime 2}\right)$ in the action which are reproduced by the corresponding disk level scattering amplitude. In particular, it has been found in [6] that the consistency with T-duality/S-matrix requires the non-constant dilaton appears in the string frame action only as the overall factor of $e^{-\phi}$.

The CS part on the other hand describes the coupling of D-branes to the RR fields. For constant background fields it is given by [7, 8]

$$
S_{C S}=T_{p} \int_{M^{p+1}} e^{B} C
$$

where $M^{p+1}$ represents the world volume of the $\mathrm{D}_{p}$-brane, $C$ is meant to represent a sum over all appropriate RR potential forms and the multiplication rule is the wedge product. The abelian gauge field can be added to the action as $B \rightarrow B+2 \pi \alpha^{\prime} F$. The curvature corrections to this action has been found by requiring that the chiral anomaly on the world volume of intersecting D-branes (I-brane) cancels with the anomalous variation of the CS action [9, 10, 11]. This correction is

$$
S_{C S}=T_{p} \int_{M^{p+1}} e^{B} C\left(\frac{\mathcal{A}\left(4 \pi^{2} \alpha^{\prime} R_{T}\right)}{\mathcal{A}\left(4 \pi^{2} \alpha^{\prime} R_{N}\right)}\right)^{1 / 2}
$$

where $\mathcal{A}\left(R_{T, N}\right)$ is the Dirac roof genus of the tangent and normal bundle curvatures respectively,

$$
\sqrt{\frac{\mathcal{A}\left(4 \pi^{2} \alpha^{\prime} R_{T}\right)}{\mathcal{A}\left(4 \pi^{2} \alpha^{\prime} R_{N}\right)}}=1+\frac{\left(4 \pi^{2} \alpha^{\prime}\right)^{2}}{384 \pi^{2}}\left(\operatorname{tr} R_{T}^{2}-\operatorname{tr} R_{N}^{2}\right)+\cdots
$$

\footnotetext{
${ }^{2}$ Our index conversion is that the Greek letters $(\mu, \nu, \cdots)$ are the indices of the space-time coordinates, the Latin letters $(a, d, c, \cdots)$ are the world-volume indices and the letters $(i, j, k, \cdots)$ are the normal bundle indices.
} 
For totally-geodesic embeddings of world-volume in the ambient spacetime, $\mathrm{R}_{T, N}$ are the pulled back curvature 2-forms of the tangent and normal bundles respectively (see the appendix in ref. [3] for more details).

It has been pointed out in [12] that the anomalous CS couplings (3) must be incomplete for non-constant B-field as they are not compatible with T-duality. T-duality exchanges the components of the metric and the B-field whereas the couplings (3) includes only the curvature terms. Compatibility of this action with T-duality should give a bunch of new couplings [13, 14].

In this paper we would like to show that for non-constant RR and NSNS fields there are other contribution to the action (3) at order $O\left(\alpha^{\prime 2}\right)$ which may not arise from requiring the consistency of the action (3) with T-duality. These terms which involve linear NSNS field can be found by studying the S-matrix element of one RR and one NSNS vertex operators [4] and by requiring them to be consistent with linear T-duality. We will find the following string frame couplings at order $O\left(\alpha^{\prime 2}\right)$ :

$$
\begin{aligned}
S \sim T_{p} \int d^{p+1} x \epsilon^{a_{0} \cdots a_{p}}( & \frac{1}{2 !(p-1) !}\left[F_{i a_{2} \cdots a_{p}, a}^{(p)} H_{a_{0} a_{1}}{ }^{a, i}-\frac{1}{p} F_{a_{1} a_{2} \cdots a_{p}, i}^{(p)}\left(H_{a_{0} a}{ }^{i, a}-H_{a_{0} j}{ }^{i, j}\right)\right] \\
& +\frac{2}{p !}\left[\frac{1}{2 !} F_{i a_{1} \cdots a_{p} j, a}^{(p+2)} \mathcal{R}^{a}{ }_{a_{0}}{ }^{i j}-\frac{1}{p+1} F_{a_{0} \cdots a_{p} j, i}^{(p+2)} \hat{\mathcal{R}}^{i j}\right] \\
& \left.-\frac{1}{3 !(p+1) !} F_{i a_{0} \cdots a_{p} j k, a}^{(p+4)} H^{i j k, a}\right)
\end{aligned}
$$

where as usual commas denote partial differentiation.

It has been shown in [6] that the compatibility of the curvature corrections to the DBI action with linear T-duality transformations requires the non-constant dilaton appears in the string frame action only through the overall factor of $e^{-\phi}$. This factor has been absorbed in the RR field so one expects that the dilaton appears in the above action only through the string frame metric. We will show that the coupling of one $F^{(p+2)}$ and one dilaton in the Einstein frame which can be calculated by the S-matrix element, is reproduced exactly by transforming the couplings in the second line above to the Einstein frame.

The couplings in (5) have been found by the S-matrix element of one RR and one NSNS vertex operators and by T-duality. The S-matrix method produces the on-shell couplings and consistency of the couplings with linear T-duality then fixes the on-shell ambiguity of the couplings. Correction to this action can also be found by requiring it to be consistent with nonlinear T-duality transformations. We will consider one particular nonlinear term in the T-duality transformation of the RR field and then examine the consistency of (5) with it to find new couplings. The new couplings are given by the above action in which $F^{(n)}$ is replaced by $\mathcal{F}^{(n)}$ where

$$
\mathcal{F}^{(n)}=F^{(n)}+B \wedge F^{(n-2)}+H \wedge C^{(n-3)}
$$




$$
\begin{aligned}
& +\frac{1}{2 !} B \wedge B \wedge F^{(n-4)}+\frac{1}{2 !} B \wedge H \wedge C^{(n-5)}+\frac{1}{2 !} H \wedge B \wedge C^{(n-5)}+\cdots \\
= & d \mathcal{C}^{(n)}
\end{aligned}
$$

where $\mathcal{C}=e^{B} C$, is the RR potential in the CS action (2).

An outline of the paper is as follows: We begin the section 2 by writing the S-matrix element of one RR and one NSNS vertex operators. From the contact terms of this amplitude at order $\left(\alpha^{\prime}\right)^{2}$, we will find the on-shell couplings of one massless RR and two NSNS fields. In section 3, we review the T-duality transformations and the strategy for checking the consistency of a D-brane action with T-duality. In section 3.1, we check the consistency of the couplings found in section 2 with linear T-duality which fixes the on-shell ambiguity of the couplings. After fixing the on-shell ambiguity of the gravity couplings, we show that the dilaton appears in the action only through the string frame metric. In section 3.2, we check the consistency of the couplings (5) with nonlinear T-duality and show that the field strength in the action (5) should be given by (6).

\section{Scattering amplitudes}

A method for finding the couplings in effective field theory is the S-matrix method. The standard CS coupling (2) has been confirmed by the S-matrix method in e.g., [15, 16]. The couplings of NSNS and RR fluxes to various types of D-branes have been found in [17] by evaluating disk amplitudes among two open string and one closed string vertex operators. To find the couplings of one RR and one NSNS states to $\mathrm{D}_{p}$-brane, one needs the scattering amplitude of their corresponding vertex operators which is given by [4]

$$
\begin{aligned}
A\left(\varepsilon_{1}, p_{1} ; \varepsilon_{2}, p_{2}\right) & =-\frac{1}{8} T_{p} \alpha^{\prime 2} K(1,2) \frac{\Gamma\left(-\alpha^{\prime} t / 4\right) \Gamma\left(\alpha^{\prime} q^{2}\right)}{\Gamma\left(1-\alpha^{\prime} t / 4+\alpha^{\prime} q^{2}\right)} \\
& =\frac{1}{2} T_{p} K(1,2)\left(\frac{1}{q^{2} t}+\frac{\pi^{2} \alpha^{\prime 2}}{24}+O\left(\alpha^{\prime 4}\right)\right)
\end{aligned}
$$

where $q^{2}=p_{1}^{a} p_{1}^{b} \eta_{a b}$ is the momentum flowing along the world-volume of D-brane, and $t=-\left(p_{1}+p_{2}\right)^{2}$ is the momentum transfer in the transverse direction. The kinematic factor is

$$
\begin{aligned}
& K(1,2)=i \frac{q^{2}}{\sqrt{2}} \operatorname{Tr}\left(P_{-} \Gamma_{1(n)} M_{p} \gamma^{\nu} \gamma \cdot\left(p_{1}+p_{2}\right) \gamma^{\mu}\right)\left(\varepsilon_{2} \cdot D\right)_{\mu \nu} \\
& -i \frac{t}{2 \sqrt{2}}\left[\operatorname{Tr}\left(P_{-} \Gamma_{1(n)} M_{p} \gamma \cdot D \cdot \varepsilon_{2}^{T} \cdot D \cdot p_{2}\right)-\operatorname{Tr}\left(P_{-} \Gamma_{1(n)} M_{p} \gamma \cdot \varepsilon_{2} \cdot D \cdot p_{2}\right)\right. \\
& \left.-\operatorname{Tr}\left(P_{-} \Gamma_{1(n)} M_{p} \gamma \cdot D \cdot p_{2}\right) \operatorname{Tr}\left(\varepsilon_{2} \cdot D\right)\right]
\end{aligned}
$$


where the matrix $D_{\nu}^{\mu}$ is diagonal with +1 in the world volume directions and -1 in the transverse directions, and

$$
\begin{aligned}
\Gamma_{1(n)} & =\frac{1}{n !} F_{1 \nu_{1} \cdots \nu_{n}} \gamma^{\nu_{1}} \cdots \gamma^{\nu_{n}} \\
M_{p} & =\frac{ \pm 1}{(p+1) !} \epsilon_{a_{0} \cdots a_{p}} \gamma^{a_{0}} \cdots \gamma^{a_{p}}
\end{aligned}
$$

where $F_{1}$ is the linearized RR field strength $n$-form and $\epsilon$ is the volume $p+1$-form of the $D_{p}$-brane. In equation (8),$P_{-}=\frac{1}{2}\left(1-\gamma_{11}\right)$ is the chiral projection operator and $\varepsilon_{2}$ is the NS-NS polarization. The $\gamma_{11}$ in the chiral projection gives the magnetic couplings and 1 gives the electric couplings. The first term in (7) produces the massless poles resulting from the $\left(\alpha^{\prime}\right)^{0}$ order of the DBI and CS couplings on the D-brane, and the supergravity couplings in the bulk. The second term in (7) should produce $\left(\alpha^{\prime}\right)^{2}$ couplings of one RR and one NSNS on the D-brane in which we are interested.

Using the identity $M_{p} \gamma^{\mu}=D^{\mu}{ }_{\nu} \gamma^{\nu} M_{p}$ [4] and the algebra $\left\{\gamma^{\mu}, \gamma^{\nu}\right\}=-2 \eta^{\mu \nu}$, one can write the above kinematic factor for the electric couplings as

$$
\begin{aligned}
K(1,2)= & i \frac{q^{2}}{2 \sqrt{2}} \operatorname{Tr}\left(\Gamma_{1(n)} \gamma^{\nu} M_{p} \gamma \cdot\left(p_{1}+p_{2}\right) \gamma^{\mu}\right)\left(\varepsilon_{2}\right)_{\mu \nu} \\
& -i \frac{t}{4 \sqrt{2}}\left[-\operatorname{Tr}\left(\Gamma_{1(n)} \gamma \cdot D \cdot \varepsilon_{2} \cdot D \cdot p_{2} M_{p}\right)\right. \\
& \left.-\frac{1}{2} \operatorname{Tr}\left(\Gamma_{1(n)} \gamma^{\mu} M_{p} \gamma \cdot D \cdot p_{2} \gamma^{\nu}\right)\left(D \cdot \varepsilon_{2} \cdot D+\varepsilon_{2}^{T}\right)_{\mu \nu}\right]
\end{aligned}
$$

One can easily check that the kinematic factor is zero for $n \leq p-2$ and for $n>p+4$. This factor is non-zero for $n=p, n=p+2$ and for $n=p+4$. Let us consider each case separately.

\section{$2.1 n=p$ case}

For $n=p$ case, one needs to perform the following traces:

$$
\operatorname{Tr}\left(\gamma^{\mu_{1}} \cdots \gamma^{\mu_{p}} \gamma^{\mu} \gamma^{a_{0}} \cdots \gamma^{a_{p}} \gamma^{\alpha} \gamma^{\nu}\right) \text { and } \operatorname{Tr}\left(\gamma^{\mu_{1}} \cdots \gamma^{\mu_{p}} \gamma^{\alpha} \gamma^{a_{0}} \cdots \gamma^{a_{p}}\right)
$$

They make various contraction of the indices. The first one simplifies to

$$
\begin{aligned}
& (-1)^{p}\left[\eta^{\mu \nu} \eta^{\alpha a_{0}} \eta^{\mu_{1} a_{1}}+\eta^{\alpha \nu} \eta^{a_{0} \mu} \eta^{a_{1} \mu_{1}}-\eta^{\alpha \mu} \eta^{a_{0} \nu} \eta^{a_{1} \mu_{1}}-p \eta^{\alpha \mu_{1}} \eta^{a_{0} \mu} \eta^{a_{1} \nu}\right. \\
& \left.-p \eta^{\alpha a_{0}} \eta^{\mu \mu_{1}} \eta^{a_{1} \nu}-p \eta^{\alpha a_{0}} \eta^{a_{1} \mu} \eta^{\nu \mu_{1}}\right] p(p+1) \operatorname{Tr}\left(\gamma^{\mu_{2}} \cdots \gamma^{\mu_{p}} \gamma^{a_{2}} \cdots \gamma^{a_{p}}\right)
\end{aligned}
$$

The second trace simplifies to

$$
\operatorname{Tr}\left(\gamma^{\mu_{1}} \cdots \gamma^{\mu_{p}} \gamma^{\alpha} \gamma^{a_{0}} \cdots \gamma^{a_{p}}\right)=(-1)^{p} \eta^{\alpha a_{0}} \eta^{\mu_{1} a_{1}} p(p+1) \operatorname{Tr}\left(\gamma^{\mu_{2}} \cdots \gamma^{\mu_{p}} \gamma^{a_{2}} \cdots \gamma^{a_{p}}\right)
$$


and the trace $\operatorname{Tr}\left(\gamma^{\mu_{2}} \cdots \gamma^{\mu_{p}} \gamma^{a_{2}} \cdots \gamma^{a_{p}}\right)$ causes the RR field strength to contract with the volume form $\epsilon$, i.e., $F_{1 \mu_{1} a_{2} \cdots a_{p}} \epsilon^{a_{0} \cdots a_{p}}$.

Using the above traces, one finds that the kinematic factor (10) for the graviton is

$$
K(1,2) \sim-i \frac{t}{2 \sqrt{2}}\left[F_{1 a_{1} \cdots a_{p}}\left(\varepsilon_{2}\right)_{a_{0}}{ }^{a} p_{2 a}+p F_{1 a a_{2} \cdots a_{p}}\left(\varepsilon_{2}\right)_{a_{1}}{ }^{a} p_{2 a_{0}}\right] \epsilon^{a_{0} \cdots a_{p}}
$$

Using the fact that the indices $a_{0}, \cdots, a_{p}$ contracted with the totally antisymmetric $\epsilon^{a_{0} \cdots a_{p}}$ tenser and the conservation of the momentum $p_{1 a}+p_{2 a}=0$, one can write

$$
\begin{aligned}
p F_{1 a a_{2} \cdots a_{p}} p_{2 a_{0}} & =p p_{1 a} C_{1 a_{2} \cdots a_{p}} p_{2 a_{0}} \\
& =F_{1 a_{0} a_{2} \cdots a_{p}} p_{2 a}
\end{aligned}
$$

which makes the kinematic factor (14) to be zero. For B-field, one finds the following non-zero result for the kinematic factor (10):

$$
\begin{aligned}
K(1,2) \sim & -\left(i \frac{q^{2}}{2 \sqrt{2}}\left[2 F_{1 a_{1} a_{2} \cdots a_{p}}\left(\varepsilon_{2}\right)_{a_{0} i}\left(p_{1}+p_{2}\right)^{i}-p F_{1 i a_{2} \cdots a_{p}}\left(\varepsilon_{2}\right)_{a_{0} a_{1}}\left(p_{1}+p_{2}\right)^{i}\right]\right. \\
& \left.-i \frac{t}{\sqrt{2}} F_{1 a_{1} a_{2} \cdots a_{p}}\left(\varepsilon_{2}\right)_{a_{0} i} p_{2}^{i}\right) \epsilon^{a_{0} a_{1} \cdots a_{p}}
\end{aligned}
$$

As a check of the calculation, if one replaces the B-field polarization with $\left(\varepsilon_{2}\right)_{\mu \nu} \rightarrow \zeta_{\mu}\left(p_{2}\right)_{\nu}-$ $\zeta_{\nu}\left(p_{2}\right)_{\mu}$ the kinematic factor vanishes, as expected from the Ward identity.

To find the field theory couplings corresponding to the above momentum space contact terms, we use the following identities:

$$
\begin{aligned}
F_{1 a_{1} \cdots a_{p}}\left(\varepsilon_{2}\right)_{a_{0} a} & =\frac{p}{2} F_{1 a a_{2} \cdots a_{p}}\left(\varepsilon_{2}\right)_{a_{0} a_{1}} \\
F_{1 a_{1} \cdots a_{p}}\left(p_{1}\right)_{i} & =p F_{1 i a_{2} \cdots a_{p}}\left(p_{1}\right)_{a_{1}}
\end{aligned}
$$

where we have used the fact that the indices $a_{0}, \cdots, a_{p}$ contracted with the totally antisymmetric $\epsilon^{a_{0} \cdots a_{p}}$ tenser. Using these identities one can write the kinematic factor as

$$
\begin{aligned}
K(1,2) \sim & -\frac{i p}{\sqrt{2}}\left(\left(\varepsilon_{2}\right)_{a_{0} a_{1}}\left[-\frac{1}{2} p_{1} \cdot V \cdot p_{1} F_{1 i a_{2} \cdots a_{p}}\left(p_{2}\right)^{i}-p_{1} \cdot N \cdot p_{2} F_{1 a a_{2} \cdots a_{p}}\left(p_{2}\right)^{a}\right]\right. \\
& \left.+p_{1} \cdot V \cdot p_{1} F_{1 i a_{2} \cdots a_{p}}\left(p_{1}\right)_{a_{1}}\left(\varepsilon_{2}\right)_{a_{0}}{ }^{i}\right) \epsilon^{a_{0} a_{1} \cdots a_{p}}
\end{aligned}
$$

which satisfies the Ward identity. The couplings corresponding to the above terms are:

$$
\frac{T_{p}}{2 !(p-1) !} \int d^{p+1} x \epsilon^{a_{0} a_{1} \cdots a_{p}}\left(F_{i a_{2} \cdots a_{p}, a}^{(p)} H_{a_{0} a_{1}}^{a, i}-F_{a a_{2} \cdots a_{p}, i}^{(p)} H_{a_{0} a_{1}}{ }^{i, a}\right)
$$

where

$$
H_{\mu \nu \alpha}=B_{\mu \nu, \alpha}+B_{\alpha \mu, \nu}+B_{\nu \alpha, \mu}
$$


The other terms in (7) correspond to the higher derivative of the couplings (19) in which we are not interested in this paper.

The last coupling in (19) has on-shell ambiguity. To see this we note that the index $a$ in this term can be either $a_{0}$ or $a_{1}$. If $a=a_{0}$, it can be written as $-2 F_{a_{0} a_{2} \cdots a_{p}, i} H_{a a_{1}} i, a / p$, and if $a=a_{1}$, it can be written as $-2 F_{a_{1} a_{2} \cdots a_{p}, i} H_{a_{0} a} a^{i, a} / p$. Interchanging $a_{1} \leftrightarrow a_{0}$ in the latter expression and using the fact that it has the overall factor of the volume form, one can write it as the former expression. Hence, the last term in (19) can be written as $-2 F_{a_{1} a_{2} \cdots a_{p}, i} H_{a_{0} a}{ }^{i, a} / p$. Moreover, using the on-shell condition $H_{\nu \rho \alpha, \mu}{ }^{\mu}=0$, one can write it as $2 F_{a_{1} a_{2} \cdots a_{p}, i} H_{a_{0} j} i, j / p$ or as

$$
-\frac{1}{p} F_{a_{1} \cdots a_{p}, i}\left(H_{a_{0} a}^{i, a}-H_{a_{0} j}^{i, j}\right)
$$

We will fix the above on-shell ambiguity in section 3 by requiring the consistency of the coupling with the T-duality transformations.

\section{$2.2 n=p+2$ case}

For $n=p+2$ case, the traces in (10) simplify to

$$
\begin{aligned}
\operatorname{Tr}\left(\gamma^{\mu_{1}} \cdots \gamma^{\mu_{p+2}} \gamma^{\mu} \gamma^{a_{0}} \cdots \gamma^{a_{p}} \gamma^{\alpha} \gamma^{\nu}\right)= & (p+1)(p+2) \operatorname{Tr}\left(\gamma^{\mu_{3}} \cdots \gamma^{\mu_{p+2}} \gamma^{a_{1}} \cdots \gamma^{a_{p}}\right) \times \\
& {\left[-\eta^{\mu \nu} \eta^{\alpha \mu_{1}} \eta^{a_{0} \mu_{2}}+\eta^{\alpha \nu} \eta^{a_{0} \mu_{2}} \eta^{\mu \mu_{1}}+\eta^{\alpha \mu} \eta^{\mu_{1} \nu} \eta^{a_{0} \mu_{2}}+\right.} \\
& \left.(p+1)\left(\eta^{\alpha \mu_{1}} \eta^{\mu_{2} \mu} \eta^{a_{0} \nu}+\eta^{\alpha \mu_{1}} \eta^{\mu a_{0}} \eta^{\mu_{2} \nu}+\eta^{\alpha a_{0}} \eta^{\mu_{1} \mu} \eta^{\nu \mu_{2}}\right)\right] \\
\operatorname{Tr}\left(\gamma^{\mu_{1}} \cdots \gamma^{\mu_{p+2}} \gamma^{\alpha} \gamma^{a_{0}} \cdots \gamma^{a_{p}}\right)= & -\eta^{\alpha \mu_{1}} \eta^{\mu_{2} a_{0}}(p+1)(p+2) \operatorname{Tr}\left(\gamma^{\mu_{3}} \cdots \gamma^{\mu_{p+2}} \gamma^{a_{1}} \cdots \gamma^{a_{p}}\right)
\end{aligned}
$$

The trace $\operatorname{Tr}\left(\gamma^{\mu_{3}} \cdots \gamma^{\mu_{p+2}} \gamma^{a_{1}} \cdots \gamma^{a_{p}}\right)$ causes the RR field strength to contract with the volume form as $F_{i \mu_{1} \mu_{2} a_{1} \cdots a_{p}} \epsilon^{a_{0} \cdots a_{p}}$. Inserting these traces in (10), one finds the kinematic factor for B-field becomes

$$
K(1,2) \sim-i \frac{t}{2 \sqrt{2}}\left[-F_{1 i a_{0} \cdots a_{p}}\left(\varepsilon_{2}\right)^{i}{ }_{a} p_{2 a}+(p+1) F_{1 i a a_{1} \cdots a_{p}}\left(\varepsilon_{2}\right)^{i}{ }_{a} p_{2 a_{0}}\right] \epsilon^{a_{0} \cdots a_{p}}
$$

Using the fact that the indices $a_{0}, \cdots, a_{p}$ contract with the totally antisymmetric tensor $\epsilon^{a_{0} \cdots a_{p}}$ and the conservation of the momentum $p_{1 a}+p_{2 a}=0$, one can write

$$
(p+1) F_{1 i a a_{1} \cdots a_{p}} p_{2 a_{0}} \ell^{a}=F_{1 i a_{0} a_{1} \cdots a_{p}} p_{2 a} \ell^{a}
$$

for any vector $\ell^{a}$. This makes the kinematic factor (22) to be zero. For the symmetric polarization, graviton or dilaton, one finds the kinematic factor (10) to be

$$
\begin{aligned}
K(1,2) \sim & \left(i \frac{q^{2}}{\sqrt{2}}\left[F_{1 j a_{0} \cdots a_{p}}\left(\varepsilon_{2}\right)^{j}{ }_{i}\left(p_{1}+p_{2}\right)^{i}+(p+1) F_{1 i \mu a_{1} \cdots a_{p}}\left(\varepsilon_{2}\right)^{\mu}{ }_{a_{0}}\left(p_{1}+p_{2}\right)^{i}\right]\right. \\
& +i \frac{t}{2 \sqrt{2}}\left[2 F_{1 i a_{0} \cdots a_{p}}\left(\varepsilon_{2}\right)^{i a} p_{2 a}-(p+1) F_{1 a a_{1} \cdots a_{p}}\left(\varepsilon_{2}\right)^{a}{ }_{a_{0}} p_{2}^{i}\right] \\
& \left.-\frac{i}{2 \sqrt{2}} F_{1 i a_{0} \cdots a_{p}} p_{2}^{i}\left(q^{2}-\frac{t}{2}\right) \operatorname{Tr}\left(\varepsilon_{2}\right)\right) \epsilon^{a_{0} a_{1} \cdots a_{p}}
\end{aligned}
$$


The last term is zero for graviton, but is has contribution to the dilaton amplitude. Using the identity (23), one can write the above equation for the graviton as

$$
\begin{aligned}
K(1,2) \sim & \frac{i(p+1)}{\sqrt{2}}\left(F_{1 j a a_{1} \cdots a_{p}} p_{2 a_{0}} p_{2}^{a}\left(\varepsilon_{2}\right)^{j}{ }_{i} p_{1}^{i}-p_{1} \cdot V \cdot p_{2} F_{1 i j a_{1} \cdots a_{p}}\left(\varepsilon_{2}\right)^{j} a_{0} p_{2}^{i}\right. \\
& \left.+p_{1} \cdot N \cdot p_{2}\left[F_{1 i a a_{1} \cdots a_{p}}\left(\varepsilon_{2}\right)^{a}{ }_{a_{0}} p_{2}^{i}-2 F_{1 i a a_{1} \cdots a_{p}}\left(\varepsilon_{2}\right)^{a i} p_{2 a_{0}}\right]\right) \epsilon^{a_{0} a_{1} \cdots a_{p}}
\end{aligned}
$$

It satisfies the Ward identity. Using the identity

$$
F_{1 j a a_{1} \cdots a_{p}} p_{1 i} p_{2 a_{0}}=F_{1 i a a_{1} \cdots a_{p}} p_{1 j} p_{2 a_{0}}-F_{1 i j a_{1} \cdots a_{p}} p_{1 a} p_{2 a_{0}}
$$

one finds that the field theory corresponding to the above amplitude is

$$
\frac{T_{p}}{p !} \int d^{p+1} x \epsilon^{a_{0} a_{1} \cdots a_{p}}\left(\frac{1}{2} F_{i j a_{1} \cdots a_{p}, a}^{(p+2)} \mathcal{R}_{a_{0}}^{a}{ }^{i j}+F_{j a a_{1} \cdots a_{p}, i}^{(p+2)} \mathcal{R}_{a_{0}}^{i}{ }^{a j}\right)
$$

The Riemann tensor at the linear order in the graviton is

$$
\mathcal{R}_{\mu \nu \rho \lambda}=\frac{1}{2}\left(h_{\mu \lambda, \nu \rho}+h_{\nu \rho, \mu \lambda}-h_{\mu \rho, \nu \lambda}-h_{\nu \lambda, \mu \rho}\right)
$$

where we have considered perturbation around the flat space where the metric takes the form $G_{\mu \nu}=\eta_{\mu \nu}+h_{\mu \nu}$. The last term in the above amplitude has again on-shell ambiguity. We will show in section 3 that this term in the present form is not consistent with T-duality. However, it can be written in a T-dual invariant form using the on-shell conditions.

The dilaton amplitude can be found from the amplitude (24) by using the following polarization:

$$
\varepsilon_{\mu \nu}=\frac{1}{\sqrt{8}}\left(\eta_{\mu \nu}-\ell_{\mu} p_{\nu}-\ell_{\nu} p_{\mu}\right) ; \quad \ell \cdot p=1
$$

where the auxiliary vector $\ell_{\mu}$ insures that the polarization satisfies the on-shell condition $p \cdot \varepsilon_{\nu}=0$. One finds the dilaton amplitude to be

$$
K(1,2) \sim \frac{i(p-3)}{\sqrt{2}}\left(p_{1} \cdot N \cdot p_{2} F_{1 i a_{0} \cdots a_{p}} p_{2}^{i}\right) \epsilon^{a_{0} a_{1} \cdots a_{p}}
$$

The field theory corresponding to the above amplitude is

$$
\frac{(p-3) T_{p}}{(p+1) !} \int d^{p+1} x \epsilon^{a_{0} a_{1} \cdots a_{p}}\left(F_{i a_{0} \cdots a_{p}, j}^{(p+2)} \phi^{, i j}\right)
$$

This coupling is zero for $\mathrm{D}_{3}$-brane which is consistent with the fact that the world volume theory of $\mathrm{D}_{3}$-brane is a conformal field theory. 


\section{$2.3 n=p+4$ case}

For $n=p+4$ case, one needs only the following trace:

$$
\begin{aligned}
& \operatorname{Tr}\left(\gamma^{\mu_{1}} \cdots \gamma^{\mu_{p+4}} \gamma^{\mu} \gamma^{a_{0}} \cdots \gamma^{a_{p}} \gamma^{\alpha} \gamma^{\nu}\right) \\
& =(-1)^{p} \eta^{\alpha \mu_{1}} \eta^{\mu \mu_{2}} \eta^{\nu \mu_{3}}(p+2)(p+3)(p+4) \operatorname{Tr}\left(\gamma^{\mu_{4}} \cdots \gamma^{\mu_{p+4}} \gamma^{a_{0}} \cdots \gamma^{a_{p}}\right)
\end{aligned}
$$

One can easily check that the kinematic factor (10) is zero for graviton, and for B-field it is

$$
K(1,2) \sim-i \frac{q^{2}}{2 \sqrt{2}} F_{1 i j k a_{0} \cdots a_{p}}\left(\varepsilon_{2}\right)^{j k} p_{2}^{i} \epsilon^{a_{0} \cdots a_{p}}
$$

which satisfies the Ward identity. The coupling corresponding to the above amplitude is

$$
\frac{T_{p}}{3 !(p+1) !} \int d^{p+1} x \epsilon^{a_{0} \cdots a_{p}} F_{i j k a_{0} \cdots a_{p}, a}^{(p+4)} H^{i j k, a}
$$

Note that $H^{i j k, a}=H^{i j a, k}$ when the indices $i, j, k$ are totally antisymmetric, as in above equation. In the next section, we will examine that the consistency of the above couplings with T-duality.

\section{T-duality}

In this section we would like to study the transformation of the couplings that we have found in the previous section under the T-duality. We denote $y$ the Killing direction along which the T-duality is going to be implemented. The full set of T-duality transformations has been found in [18, 19]

$$
\begin{aligned}
e^{2 \tilde{\phi}} & =\frac{e^{2 \phi}}{G_{y y}} \\
\tilde{G}_{y y} & =\frac{1}{G_{y y}} \\
\tilde{G}_{\mu y} & =\frac{B_{\mu y}}{G_{y y}} \\
\tilde{G}_{\mu \nu} & =G_{\mu \nu}-\frac{G_{\mu y} G_{\nu y}-B_{\mu y} B_{\nu y}}{G_{y y}} \\
\tilde{B}_{\mu y} & =\frac{G_{\mu y}}{G_{y y}} \\
\tilde{B}_{\mu \nu} & =B_{\mu \nu}-\frac{B_{\mu y} G_{\nu y}-G_{\mu y} B_{\nu y}}{G_{y y}}
\end{aligned}
$$




$$
\begin{aligned}
& \tilde{C}_{\mu \cdots \nu \alpha y}^{(n)}=C_{\mu \cdots \nu \alpha}^{(n-1)}-(n-1) \frac{C_{[\mu \cdots \nu \mid y}^{(n-1)} G_{\mid \alpha] y}}{G_{y y}} \\
& \tilde{C}_{\mu \cdots \nu \alpha \beta}^{(n)}=C_{\mu \cdots \nu \alpha \beta y}^{(n+1)}+n C_{[\mu \cdots \nu \alpha}^{(n-1)} B_{\beta] y}+n(n-1) \frac{C_{[\mu \cdots \nu \mid y}^{(n-1)} B_{|\alpha| y} G_{\mid \beta] y}}{G_{y y}}
\end{aligned}
$$

where $\mu, \nu, \alpha, \beta \neq y$. In above transformation the metric is the string frame metric. If $y$ is identified on a circle of radius $R$, i.e., $y \sim y+2 \pi R$, then after T-duality the radius becomes $\tilde{R}=\alpha^{\prime} / R$. The string coupling is also shifted as $\tilde{g}=g \sqrt{\alpha^{\prime}} / R$.

The strategy for finding T-duality invariant couplings is given in [6]. Let us review it here. Suppose we are implementing T-duality along a world volume direction of a $\mathrm{D}_{p}$-brane denoted $y$. One should first separate the world-volume indices into $y$ index and the worldvolume indices which do not include $y$, and then apply the above T-duality transformations. The latter indices are complete world-volume indices of the T-dual $\mathrm{D}_{p-1}$-brane. However, the $y$ index in the T-dual theory which is a normal bundle index is not a complete index. On the other hand, the normal bundle indices of the original theory are not complete in the T-dual $\mathrm{D}_{p-1}$-brane. They are not include $y$. In a T-duality invariant theory, the $y$ indices must be combined with the incomplete normal bundle indices to give the complete normal bundle indices in the T-dual $\mathrm{D}_{p-1}$-brane. If a theory is not invariant under the T-duality, one should then add new terms to it to have the complete indices in the T-dual theory. In this way one makes the theory to be T-duality invariant by adding new couplings.

One may also implement T-duality along a transverse direction of a $\mathrm{D}_{p}$-brane denoted $y$. In this case one must first separate the transverse indices to $y$ and the transverse indices which do not include $y$, and then apply the above T-duality transformations. The latter indices are the complete transverse indices of the $\mathrm{T}$-dual $\mathrm{D}_{p+1}$-brane. However, the complete world-volume indices of the original $\mathrm{D}_{p}$-brane are not the complete indices of the T-dual $\mathrm{D}_{p+1}$-brane. They must include the $y$ index to be complete. In a T-duality invariant theory, the $y$ index which is a world-volume index in the T-dual theory must be combined

with the incomplete world-volume indices of the $\mathrm{T}$-dual $\mathrm{D}_{p+1}$-brane to give the complete world-volume indices.

\subsection{Linear T-duality}

In this subsection we would like to study the consistency of the couplings with linear Tduality transformations. Assuming the NSNS and RR fields are small perturbations around the flat space, the T-duality transformations take the following linear form:

$$
\begin{aligned}
& \tilde{\phi}=\phi-\frac{1}{2} h_{y y}, \tilde{h}_{y y}=-h_{y y}, \tilde{h}_{\mu y}=B_{\mu y}, \tilde{B}_{\mu y}=h_{\mu y}, \tilde{h}_{\mu \nu}=h_{\mu \nu}, \tilde{B}_{\mu \nu}=B_{\mu \nu} \\
& \tilde{C}_{\mu \cdots \nu \alpha y}^{(n)}=C_{\mu \cdots \nu \alpha}^{(n-1)}, \tilde{C}_{\mu \cdots \nu \alpha \beta}^{(n)}=C_{\mu \cdots \nu \alpha \beta y}^{(n+1)}
\end{aligned}
$$


Consistency of the curvature squared corrections to the DBI action under the above linear T-duality transformations has been examined in [6]. The consistency requires adding some $H$-squared terms to the DBI action which are also consistent with the corresponding Smatrix element. We are going to do similar calculation for the couplings that we have found in the previous section.

We begin by studying the T-duality of the couplings in (19). Consider implementing T-duality along a world volume direction of the $\mathrm{D}_{p}$-brane 3 . From the contraction with the world volume form, one of the indices $a_{2}, \cdots, a_{p}$ of the RR field strength or the indices $a_{0}, a_{1}$ of the NSNS field strength in (19) must include $y$, and so there are two cases to consider: First when $y$ appears as an index on the RR field strength and second when $y$ is an index in the NSNS field strength. In the former case, we note that there are $p-1$ indices in the RR field strength which are contracted with the volume form. Each of these indices can be $y$. However, because of the totally antisymmetric property of the volume form and the RR field strength, they all are identical. So one can write (19) as

$$
\frac{T_{p}}{2 !(p-2) !} \int d^{p+1} x \epsilon^{a_{0} a_{1} \cdots a_{p-1} y}\left(F_{i a_{2} \cdots a_{p-1} y, a}^{(p)} H_{a_{0} a_{1}}^{a, i}-F_{a a_{2} \cdots a_{p-1} y, i}^{(p)} H_{a_{0} a_{1}}{ }^{i, a}\right)
$$

Note that the indices $i, a$ appear as the derivative indices so nigher of them can be $y$. Moreover, because of the world volume form, none of the indices $a_{0}, \cdots a_{p-1}$ can be $y$. The transform of the above couplings under the linear T-duality (36) gives the following couplings for $\mathrm{D}_{p-1}$-brane:

$$
2 \pi \sqrt{\alpha^{\prime}} \frac{T_{p}}{2 !(p-2) !} \int d^{p} x \epsilon^{a_{0} a_{1} \cdots a_{p-1}}\left(F_{i a_{2} \cdots a_{p-1}, a}^{(p-1)} H_{a_{0} a_{1}}^{a, i}-F_{a a_{2} \cdots a_{p-1}, i}^{(p-1)} H_{a_{0} a_{1}}{ }^{i, a}\right)
$$

where we have also used the fact that $T_{p} \sim 1 / g_{s}$. Using the relation $2 \pi \sqrt{\alpha^{\prime}} T_{p}=T_{p-1}$, one observes that the above couplings are exactly the couplings (19) for $\mathrm{D}_{p-1}$-brane.

We will now check the case that the T-dual coordinate $y$ is carried by the NSNS field strength. There are two possibilities for the NSNS field strength in (19) to carry the Tdual coordinate $y$, i.e., either $a_{0}$ or $a_{1}$ carries the index $y$. Since the two possibilities are identical, one can write (19) as

$$
\frac{T_{p}}{(p-1) !} \int d^{p+1} x \epsilon^{y a_{1} \cdots a_{p}}\left(F_{i a_{2} \cdots a_{p}, a}^{(p)} H_{y a_{1}}{ }^{a, i}-F_{a a_{2} \cdots a_{p}, i}^{(p)} H_{y a_{1}}{ }^{i, a}\right)
$$

Note again that the indices $i, a$ and $a_{1}, \cdots, a_{p}$ can not be $y$. The above couplings transform under linear T-duality to the following couplings of $\mathrm{D}_{p-1}$-brane:

$$
\frac{T_{p-1}}{(p-1) !} \int d^{p} x \epsilon^{a_{1} \cdots a_{p}}\left(2 F_{i a_{2} \cdots a_{p} y, a}^{(p+1)} \mathcal{R}_{a_{1}}^{a}{ }^{i y}-2 F_{a a_{2} \cdots a_{p} y, i}^{(p+1)} \mathcal{R}_{a_{1}}^{i}{ }^{a y}\right)
$$

\footnotetext{
${ }^{3}$ The couplings (19) are consistent with the linear T-duality transformations (36) when implementing the T-duality along a transverse direction.
} 
where we have used the assumption in T-duality that all field are independent of the Killing direction $y$. The coordinate $y$ in the T-dual theory is a transverse coordinate. Inspired by the above couplings, one may guess that the correct form of the couplings for $\mathrm{D}_{p-1}$-brane are in fact,

$$
\frac{T_{p-1}}{(p-1) !} \int d^{p} x \epsilon^{a_{1} \cdots a_{p}}\left(F_{i a_{2} \cdots a_{p} j, a}^{(p+1)} \mathcal{R}_{a_{1}}^{a}{ }^{i j}-2 F_{a a_{2} \cdots a_{p} j, i}^{(p+1)} \mathcal{R}_{a_{1}}^{i}{ }^{a j}\right)
$$

This is consistent with the couplings (27) that we have found from the S-matrix.

The last term above is not consistent with the T-duality along the world volume direction. To see this, consider the case that $a_{1}$ carries the index $y$. The world volume index $a$ should be separated into $y$ and $\tilde{a}$, which does not include the coordinate $y$. So the second term in (40) can be written as

$$
-\frac{2 T_{p-1}}{(p-1) !} \int d^{p} x \epsilon^{y a_{2} \cdots a_{p}}\left(F_{\tilde{a} a_{2} \cdots a_{p} j, i}^{(p+1)} \mathcal{R}_{y}^{i{ }_{a} j}+F_{y a_{2} \cdots a_{p} j, i}^{(p+1)} \mathcal{R}_{y}^{i}{ }^{y j}\right)
$$

Under the linear T-duality it transforms to

$$
-\frac{T_{p-1}}{(p-1) !} \int d^{p-1} x \epsilon^{a_{2} \cdots a_{p}}\left(F_{\tilde{a} a_{2} \cdots a_{p} j y, i}^{(p+2)} H^{\tilde{a} y j, i}-(-1)^{p} F_{a_{2} \cdots a_{p} j, i}^{(p)} h_{y y}{ }^{, i j}\right)
$$

The first term above, in particular, indicates that there must be the following coupling:

$$
-\frac{T_{p}}{2 !(p+1) !} \int d^{p+1} x \epsilon^{a_{0} \cdots a_{p}} F_{a a_{0} \cdots a_{p} j k, i}^{(p+4)} H^{a k j, i}
$$

However, this coupling is not produced by the S-matrix element (33). To fix this inconsistency, we use the on-shell conditions to rewrite the second term in (40) in a T-dual form. The index $a$ in this term can be only $a_{1}$ so we can rewrite it as $-2 F_{a_{1} a_{2} \cdots a_{p} j, i}^{(p+1)} \mathcal{R}_{a}^{i}{ }_{a j}{ }^{a j} / p$. Moreover, using the on-shell conditions, one can write the curvature as $\hat{\mathcal{R}}^{i j}$ where

$$
\hat{\mathcal{R}}_{i j} \equiv \frac{1}{2}\left(\mathcal{R}_{i a}{ }^{a}{ }_{j}-\mathcal{R}_{i k}{ }^{k}{ }_{j}\right)
$$

It does not have $a_{1}$ index anymore to produce inconsistency with T-duality. It has been shown in [6] that it is invariant under linear T-duality transformations (36) when $i, j \neq y$. Hence, the couplings (40) can be written for $\mathrm{D}_{p}$-brane as

$$
2 T_{p} \int d^{p+1} x \epsilon^{a_{0} \cdots a_{p}}\left(\frac{1}{2 ! p !} F_{i a_{1} \cdots a_{p} j, a}^{(p+2)} \mathcal{R}_{a_{0}}^{a}{ }^{i j}-\frac{1}{(p+1) !} F_{a_{0} \cdots a_{p} j, i}^{(p+2)} \hat{\mathcal{R}}^{i j}\right)
$$

which are equivalent to the couplings (27) using on-shell conditions. These are the couplings in the second line of (5). 
It has been speculated in [6] that the non-constant dilaton appears in the string frame D-brane action in the same way that the constant dilaton appears in the action, e.g., the non-constant dilaton appears only through the overall factor of $e^{-\phi}$ in the string frame DBI action. This proposal has been verified for DBI action by explicit calculation at order $\alpha^{2}$ in [6]. We now check the proposal for the couplings that we have found. According to this proposal the dilaton appears only through the string frame metric in (45). In other words, the dilaton couplings in the Einstein frame should be given by transforming the string frame couplings (45) to the Einstein frame, i.e., replacing $h_{\mu \nu} \rightarrow \phi \eta_{\mu \nu} / 2$. This replacement gives $\mathcal{R}^{a}{ }_{a_{0}}{ }^{i j} \rightarrow 0, \mathcal{R}^{i}{ }_{a}{ }^{a j} \rightarrow\left(\eta^{i j} \phi_{, a}{ }^{a}+\eta_{a}{ }^{a} \phi^{i j}\right) / 4$ and $\mathcal{R}_{k}{ }_{k}{ }^{k j} \rightarrow\left[\eta^{i j} \phi_{, k}{ }^{k}+\left(\eta_{k}{ }^{k}-2\right) \phi^{, i j}\right] / 4$. Using the on-shell condition that $F_{a_{0} \cdots a_{p} i}{ }^{i}=-F_{a_{0} \cdots a_{p} a}{ }^{a}=0$, one finds the following dilaton coupling:

$$
-\frac{T_{p}(p-3)}{2(p+1) !} \int d^{p+1} x \epsilon^{a_{0} \cdots a_{p}} F_{a_{0} \cdots a_{p} j, i}^{(p+2)} \phi^{, i j}
$$

which is exactly the coupling (31). Note that if one uses the replacement $h_{\mu \nu} \rightarrow \phi \eta_{\mu \nu} / 2$ in the couplings (27) which is consistent with S-matrix but not with T-duality, one would not find the correct dilaton coupling in the Einstein frame.

Having fixed the on-shell ambiguity of the last term in (27) by requiring the consistency with linear T-duality, we now fix the on-shell ambiguity of the last term in (19) by requiring that the T-duality along a transverse direction of the equation (45) should produce the $F^{(p)} H$ couplings. Reversing the steps to find the first term in (45), one finds the first term in (19). To find the T-dual of the last term in (45), we write it as

$$
-\frac{2 T_{p}}{(p+1) !} \int d^{p+1} x \epsilon^{a_{0} \cdots a_{p}}\left(F_{a_{0} \cdots a_{p} \tilde{j}, i}^{(p+2)} \tilde{\mathcal{R}}^{i \tilde{j}}+F_{a_{0} \cdots a_{p} y, i}^{(p+2)} \tilde{\mathcal{R}}^{i y}\right)
$$

where the transverse index $\tilde{j}$ does not include $y$. The T-duality transformation of the first term above gives a term which is reproduced by the second term in (45), and the T-duality of the second term gives the following terms:

$$
\frac{T_{p+1}}{2(p+1) !} \int d^{p+1} x d y \epsilon^{y a_{0} \cdots a_{p}} F_{a_{0} \cdots a_{p}, i}^{(p+1)}\left(H^{i a}{ }_{y, a}-H^{i j}{ }_{y, j}\right)
$$

Inspired by these terms, one guesses that there must be the following couplings:

$$
\frac{T_{p}}{2 p !} \int d^{p+1} x \epsilon^{a_{1} \cdots a_{p}} F_{a_{1} \cdots a_{p}, i}^{(p+1)}\left(H^{i a}{ }_{a_{0}, a}-H^{i j}{ }_{a_{0}, j}\right)
$$

This fixes the on-shell ambiguity in the second term in (19). Hence, the couplings which are consistent with the S-matrix element and with the linear T-duality are those that appear in the first line of (5).

Now we consider the transformation of the couplings (45) under linear T-duality transformations along a world volume direction. From the contraction with the world volume 
form, one of the indices of the RR field strength or the index of the curvature in (45) must include $y$, and so again there are two cases to consider: First when $y$ appears as an index on the RR field strength and second when $y$ is an index in the curvature. In the former case, one can easily check that the T-dual couplings are consistent with (45). In the latter case, we write (45) as

$$
2 T_{p} \int d^{p+1} x \epsilon^{y a_{1} \cdots a_{p}}\left(\frac{1}{2 ! p !} F_{i a_{1} \cdots a_{p} j, a}^{(p+2)} \mathcal{R}_{y}^{a}{ }_{y}^{i j}\right)
$$

It transforms under linear T-duality to the following coupling of $\mathrm{D}_{p-1}$-brane:

$$
T_{p-1} \int d^{p} x \epsilon^{a_{1} \cdots a_{p}}\left(\frac{1}{2 ! p !} F_{i a_{1} \cdots a_{p} j y, a}^{(p+3)} H^{i y j, a}\right)
$$

Note that after T-duality the transverse indices $i, j$ should be written as $\tilde{i}, \tilde{j}$ which do not include $y$, however, because $H^{\tilde{i} y \tilde{i}}$ is totally antisymmetric we wrote it as $H^{i y j}$. Inspired by this couplings, one may guess that the correct form of the coupling for $\mathrm{D}_{p}$-brane is in fact,

$$
T_{p} \int d^{p+1} x \epsilon^{a_{0} a_{1} \cdots a_{p}}\left(\frac{1}{3 !(p+1) !} F_{i a_{0} \cdots a_{p} j k, a}^{(p+4)} H^{i k j, a}\right)
$$

which is the coupling (34) that we have found from the S-matrix element. This is the coupling in the last line of (5). There is no index in the NSNS field strength in above coupling that contracts with the world volume form. So continuing the T-duality along a world volume direction, one would find no new term involving $F^{p+6}$. This is consistent with the S-matrix calculation in the previous section that indicates there is no coupling for $F^{p+6}$.

\subsection{Non-linear T-duality}

We have seen in the previous section that the couplings (5) are consistent with the linear T-duality transformations (36). However, they are not consistent with nonlinear T-duality transformations. In this paper, we would like to study the effect of the second term in the T-duality transformation of the RR potential in (35). So we consider the following T-duality transformations:

$$
\tilde{h}_{\mu y}=B_{\mu y}, \tilde{B}_{\mu y}=h_{\mu y}, \tilde{h}_{\mu \nu}=h_{\mu \nu}, \tilde{B}_{\mu \nu}=B_{\mu \nu}, \tilde{C}_{\mu \cdots \nu \alpha \beta}^{(n)}=n C_{[\mu \cdots \nu \alpha}^{(n-1)} B_{\beta] y}
$$

The reason for choosing only the nonlinear term above is that the consistency of the RR potential $C$ with this term makes it to be $e^{B} C$. To see this, consider the linear coupling of the CS action (2), i.e.,

$$
T_{p} \int C^{(p+1)}=\frac{T_{p}}{(p+1) !} \int d^{p+1} x \epsilon^{a_{0} \cdots a_{p}} C_{a_{0} \cdots a_{p}}^{(p+1)}
$$


The transformation of the above coupling of $\mathrm{D}_{p}$-brane under $\mathrm{T}$-duality along a transverse direction gives the following coupling for $\mathrm{D}_{p+1}$-brane:

$$
\frac{T_{p+1}}{p !} \int d^{p+1} x d y \epsilon^{a_{0} \cdots a_{p} y} C_{a_{0} \cdots a_{p-1}}^{(p)} B_{a_{p} y}
$$

This dictates that there must be the following coupling:

$$
\frac{T_{p}}{2 !(p-1) !} \int d^{p+1} x \epsilon^{a_{0} \cdots a_{p}} C_{a_{0} \cdots a_{p-2}}^{(p-1)} B_{a_{p-1} a_{p}}=T_{p} \int C^{(p-1)} \wedge B
$$

which is a standard term in the CS action (2).

We now study the consistency of the couplings in the first line of (5) under the Tduality transformation (53). To study the effect of last term in (53), we have to consider the couplings in which the RR field carries no index $y$. We begin by implementing T-duality along a transverse direction of the $\mathrm{D}_{p}$-brane. The B-fields in the first line of (5) are invariant under (53), so these couplings transform under the T-duality to the following couplings for $\mathrm{D}_{p+1}$-brane:

$$
\frac{T_{p+1}}{2 !(p-1) !} \int d^{p+1} x d y \epsilon^{a_{0} a_{1} \cdots a_{p} y}\left(\tilde{F}_{i a_{2} \cdots a_{p}, a}^{(p)} H_{a_{0} a_{1}}{ }^{a, i}-\frac{1}{p} \tilde{F}_{a_{1} \cdots a_{p}, i}^{(p)}\left(H_{a_{0} a}{ }^{i, a}-H_{a_{0} j}{ }^{i, j}\right)\right)
$$

Let us first consider the second term above. The transformation of the RR field strength $\tilde{F}_{a_{1} \cdots a_{p}}^{(p)}$ under the T-duality (53) is

$$
\begin{aligned}
\tilde{F}_{a_{1} \cdots a_{p}}^{(p)} & =p\left(\tilde{C}_{a_{2} \cdots a_{p}}^{(p-1)}\right)_{a_{1}} \\
& =p F_{a_{1} \cdots a_{p-1}}^{(p-1)} B_{a_{p} y}+\frac{p(p-1)}{2} C_{a_{2} \cdots a_{p-1}}^{(p-2)} H_{a_{p} y a_{1}}
\end{aligned}
$$

where here and in the subsequent identities we have used the fact that the world volume indices $a_{0}, a_{1}, \cdots a_{p}$ are contracted with the totally antisymmetric world volume tensor. Inserting this in the second term in (57), one finds that there must be the following couplings:

$$
\begin{aligned}
-\frac{T_{p+1}}{2 !} \int d^{p+1} x d y \epsilon^{a_{0} a_{1} \cdots a_{p+1}} & \left(\frac{1}{2 !(p-1) !} F_{a_{1} \cdots a_{p-1}}^{(p-1)} B_{a_{p} a_{p+1}}+\frac{1}{3 !(p-2) !} C_{a_{2} \cdots a_{p-1}}^{(p-2)} H_{a_{p} a_{p+1} a_{1}}\right)_{, i} \\
& \times\left(H_{a_{0} a}^{i, a}-H_{a_{0} j}{ }^{i, j}\right)
\end{aligned}
$$

The terms in the bracket in the first line is

$$
\frac{1}{(p+1) !}\left(B \wedge F^{(p-1)}\right)_{a_{1} \cdots a_{p+1}}+\frac{1}{(p+1) !}\left(H \wedge C^{(p-2)}\right)_{a_{1} \cdots a_{p+1}}
$$

Hence, the couplings (59) are given by the second term in (5) in which the RR field strength is (6). 
The transformation of the RR field strength $\tilde{F}_{i a_{2} \cdots a_{p}}^{(p)}$ in the first term of (57) is

$$
\begin{aligned}
\tilde{F}_{i a_{2} \cdots a_{p}}^{(p)}= & \tilde{C}_{a_{2} \cdots a_{p}, i}^{(p-1)}-(p-1) \tilde{C}_{i a_{3} \cdots a_{p}, a_{2}}^{(p-1)} \\
= & (p-1) F_{i a_{2} \cdots a_{p-1}}^{(p-1)} B_{a_{p} y}+(-1)^{p-2} F_{a_{2} \cdots a_{p}}^{(p-1)} B_{i y} \\
& +(-1)^{p-2} \frac{(p-1)(p-2)}{2} C_{i a_{2} \cdots a_{p-2}}^{(p-2)} H_{a_{p-1} a_{p} y}+(p-1) C_{a_{2} \cdots a_{p-1}}^{(p-2)} H_{a_{p} y i}
\end{aligned}
$$

Inserting this in equation (57), one finds that there must be the following new terms:

$$
\begin{aligned}
& \frac{T_{p+1}}{2 !} \int d^{p+1} x d y \epsilon^{a_{0} a_{1} \cdots a_{p} a_{p+1}} H_{a_{0} a_{1}}^{a, i}\left(\frac{1}{2 !(p-2) !} F_{i a_{2} \cdots a_{p-1}}^{(p-1)} B_{a_{p} a_{p+1}}+\frac{1}{(p-1) !} F_{a_{3} \cdots a_{p+1}}^{(p-1)} B_{i a_{2}}\right. \\
& \left.-\frac{1}{3 !(p-3) !} C_{a_{2} \cdots a_{p-2} i}^{(p-2)} H_{a_{p-1} a_{p} a_{p+1}}+\frac{1}{2 !(p-2) !} C_{a_{2} \cdots a_{p-1}}^{(p-2)} H_{a_{p} a_{p+1} i}\right)_{, a}
\end{aligned}
$$

Consider the following identities:

$$
\begin{aligned}
\frac{1}{p !}\left(B \wedge F^{(p-1)}\right)_{i a_{2} \cdots a_{p+1}} & =\frac{1}{(p-1) !} B_{i a_{2}} F_{a_{3} \cdots a_{p+1}}^{(p-1)}-\frac{1}{2 !(p-2) !} B_{a_{3} a_{2}} F_{i a_{4} \cdots a_{p+1}}^{(p-2)} \\
\frac{1}{p !}\left(H \wedge C^{(p-2)}\right)_{i a_{2} \cdots a_{p+1}} & =\frac{1}{2 !(p-1) !} H_{i a_{2} a_{3}} C_{a_{4} \cdots a_{p+1}}^{(p-2)}-\frac{1}{3 !(p-3) !} H_{a_{2} a_{3} a_{4}{ }_{2}} C_{i a_{5} \cdots a_{p+1}}^{(p-2)}
\end{aligned}
$$

The sum of the above terms gives exactly the terms in the bracket in (62). Hence the new terms (62) are given by the coupling in the first term of (5) in which the RR field strength is given by (6) .

Now consider T-duality of the couplings in the first line of (5) along a world volume direction. From the contraction with the world volume form, one of the indices $a_{0}, a_{1}$ of the NSNS field strength must include $y$. Note that the RR field has no $y$ index in the nonlinear T-duality transformation (53). The T-duality on the B-field is the same as in the previous section, so the couplings transform under the T-duality (53) to the following couplings for $\mathrm{D}_{p-1}$-brane:

$$
2 \frac{T_{p-1}}{(p-1) !} \int d^{p} x \epsilon^{a_{1} \cdots a_{p}}\left(\tilde{F}_{i a_{2} \cdots a_{p}, a}^{(p)} \mathcal{R}_{a_{1}}^{a}{ }^{i y}-\frac{1}{p} \tilde{F}_{a_{1} \cdots a_{p}, i}^{(p)} \hat{\mathcal{R}}^{i y}\right)
$$

The transformation of the RR field strengths are given by (61) and (58). However, the $y$ coordinate is now a transverse coordinate, so unlike the previous case the new terms inspired by the above couplings can not be written as $B \wedge F+H \wedge C$. This indicates that there must be some other contributions as well. The other contributions are coming from the T-duality transformation of the couplings in the second line of (5) along a transverse direction. The transformation of these terms under (53) gives the following couplings for $\mathrm{D}_{p+1}$-brane:

$$
2 \frac{T_{p+1}}{p !} \int d^{p} x d y \epsilon^{a_{0} \cdots a_{p} y}\left(\frac{1}{2 !} \tilde{F}_{i a_{1} \cdots a_{p} j, a}^{(p+2)} \mathcal{R}^{a}{ }_{a_{0}}{ }^{i j}-\frac{1}{p+1} \tilde{F}_{a_{0} \cdots a_{p} j, i}^{(p+2)} \hat{\mathcal{R}}^{i j}\right)
$$


The new couplings inspired by the couplings (64) and (65) should be given by the couplings in the second line of (5) in which the RR field strength is (6)

Similarly, the T-duality of the couplings in the second line of (5) along a world volume direction is given by the following coupling for $\mathrm{D}_{p-1}$-brane:

$$
T_{p-1} \int d^{p} x \epsilon^{a_{1} \cdots a_{p}}\left(\frac{1}{2 ! p !} \tilde{F}_{i a_{1} \cdots a_{p} j, a}^{(p+2)} H^{i y j, a}\right)
$$

and the T-duality of the coupling in the last line of (5) along a transverse direction is given by the following coupling for $\mathrm{D}_{p+1}$-brane:

$$
T_{p+1} \int d^{p+1} x d y \epsilon^{a_{0} \cdots a_{p} y}\left(\frac{1}{3 !(p+1) !} \tilde{F}_{i a_{0} \cdots a_{p} j k, a}^{(p+4)} H^{i k j, a}\right)
$$

The new couplings inspired by the couplings (66) and (67) should be given by the couplings in the last line of (5) in which the RR field strength is (6). Let us check this case explicitly.

Shifting $p \rightarrow p+1$ in (66), one can write this equation as

$$
T_{p} \int d^{p+1} x \epsilon^{a_{0} \cdots a_{p}}\left(\frac{1}{2 !(p+1) !} \tilde{F}_{i a_{0} \cdots a_{p} j, a}^{(p+3)} H^{i y j, a}\right)
$$

The transformation of the RR field strength $\tilde{F}_{i a_{0} \cdots a_{p} j}^{(p+3)}$ under (53) is

$$
\begin{aligned}
\tilde{F}_{i a_{0} \cdots a_{p} j}^{(p+3)}= & 2 \tilde{C}_{a_{0} \cdots a_{p} j, i}^{(p+2)}-(p+1) \tilde{C}_{i a_{1} \cdots a_{p} j, a_{0}}^{(p+2)} \\
= & 2 F_{i a_{0} \cdots a_{p}}^{(p+2)} B_{j y}-(p+1) F_{i a_{0} \cdots a_{p-1} j}^{(p+2)} B_{a_{p} y} \\
& -2(p+1) C_{a_{0} \cdots a_{p-1} j}^{(p+1)} H_{a_{p} y i}+C_{a_{0} \cdots a_{p}}^{(p+1)} H_{j y i}+\frac{p(p+1)}{2} C_{a_{0} \cdots a_{p-2} i j}^{(p+1)} H_{a_{p} y a_{p-1}}
\end{aligned}
$$

Inserting this in equation ( 68 ), one finds that there should be the following new terms:

$$
\begin{aligned}
& T_{p} \int d^{p+1} x \epsilon^{a_{0} \cdots a_{p}}\left(\left[\frac{1}{2 !(p+1) !} F_{i a_{0} \cdots a_{p}}^{(p+2)} B_{j k}-\frac{1}{2 ! p !} F_{i a_{0} \cdots a_{p-1} j}^{(p+2)} B_{a_{p} k}\right.\right. \\
& \left.\left.-\frac{1}{2 ! p !} C_{a_{0} \cdots a_{p-1} j}^{(p+1)} H_{a_{p} k i}+\frac{1}{3 !(p+1) !} C_{a_{0} \cdots a_{p}}^{(p+1)} H_{j k i}+\frac{1}{2 ! 2 !(p-1) !} C_{a_{0} \cdots a_{p-2} i j}^{(p+1)} H_{a_{p-1} a_{p} k}\right]_{, a} H^{i k j, a}\right)
\end{aligned}
$$

Checking the indices, one realizes that the above terms are not given by the last term of (5) in which $F$ is replaced by (66). Now shifting $p \rightarrow p-1$ in equation (67), one can write it as

$$
T_{p} \int d^{p} x d y \epsilon^{a_{0} \cdots a_{p-1} y}\left(\frac{1}{3 !(p) !} \tilde{F}_{i a_{0} \cdots a_{p-1} j k, a}^{(p+3)} H^{i k j, a}\right)
$$


The transformation of the RR field strength $\tilde{F}_{i a_{0} \cdots a_{p-1} j k}^{(p+3)}$ under (53) is

$$
\begin{aligned}
\tilde{F}_{i a_{0} \cdots a_{p-1} j k}^{(p+3)}= & 3 \tilde{C}_{a_{0} \cdots a_{p-1} j k, i}^{(p+2)}-p \tilde{C}_{i a_{1} \cdots a_{p-1} j k, a_{0}}^{(p+2)} \\
= & 3 F_{i a_{0} \cdots a_{p-1} j}^{(p+2)} B_{k y}+p F_{i a_{0} \cdots a_{p-2} j k}^{(p+2)} B_{a_{p-1} y} \\
& +3 p C_{a_{0} \cdots a_{p-2} j k}^{(p+1)} H_{a_{p-1} y i}+3 C_{a_{0} \cdots a_{p-1} j}^{(p+1)} H_{k y i}+\frac{p(p-1)}{2} C_{i a_{1} \cdots a_{p-3} a_{0} j k}^{(p+1)} H_{a_{p-2} a_{p-1} y}
\end{aligned}
$$

Inserting this in equation (71), one finds that the first, third and fourth terms above are reproduced by the new couplings (70) when one chooses $a_{p}=y$ in (70). The other two terms led us to guess that there should be the following new terms:

$$
\begin{aligned}
T_{p} \int d^{p+1} x \epsilon^{a_{0} \cdots a_{p}}( & {\left[\frac{1}{2 ! 3 !(p-1) !} F_{i a_{0} \cdots a_{p-2} j k}^{(p+2)} B_{a_{p-1} a_{p}}\right.} \\
& \left.\left.-\frac{1}{3 ! 3 !(p-2) !} C_{a_{0} \cdots a_{p-3} i j k}^{(p+1)} H_{a_{p-2} a_{p-1} a_{p}}\right]_{, a} H^{i k j, a}\right)
\end{aligned}
$$

One can easily check that the new couplings (70) and (722) are reproduced by the last term in (5) in which the RR field strength is given by $B \wedge F^{(p+2)}+H \wedge C^{(p+1)}$.

Now consider the new couplings in (5) which have one RR and two NSNS states. If one implements the T-duality on these terms and use the nonlinear T-duality transformations (53), one should find new terms which are given by (5) in which the RR field strength is given by the terms in the second line of (6) . Let us check this for the second term in (5) which is simple to analyze. Implementing the T-duality along a transverse direction, one finds the following couplings for $\mathrm{D}_{p+1}$-brane:

$$
\begin{aligned}
& -\frac{T_{p+1}}{2} \int d^{p+1} x d y \epsilon^{a_{0} \cdots a_{p} y}\left(H_{a_{0} a}^{i, a}-H_{a_{0} j}{ }^{i, j}\right) \\
& \times\left(\frac{1}{2 !(p-2) !} B_{a_{1} a_{2}} \tilde{F}_{a_{3} \cdots a_{p}}^{(p-2)}+\frac{1}{3 !(p-4) !} H_{a_{1} a_{2} a_{3}} C_{a_{4} \cdots a_{p-1}}^{(p-4)} B_{a_{p} y}\right)_{, i}
\end{aligned}
$$

where the T-duality of the RR field strength is given in (58). Inspired by the above equation, one guesses that there must be the following couplings:

$$
\begin{aligned}
& -\frac{T_{p+1}}{2} \int d^{p+1} x d y \epsilon^{a_{0} \cdots a_{p} y}\left(H_{a_{0} a}^{i, a}-H_{a_{0} j}{ }^{i, j}\right)\left(\frac{1}{2 ! 2 ! 2 !(p-3) !} B_{a_{1} a_{2}} F_{a_{3} \cdots a_{p-1}}^{(p-3)} B_{a_{p} a_{p+1}}\right. \\
& \left.+\frac{1}{2 ! 2 ! 3 !(p-4) !} B_{a_{1} a_{2}} C_{a_{4} \cdots a_{p-1}}^{(p-4)} H_{a_{p} a_{p+1} a_{3}}+\frac{1}{2 ! 2 ! 3 !(p-4) !} H_{a_{1} a_{2} a_{3}} C_{a_{4} \cdots a_{p-1}}^{(p-4)} B_{a_{p} a_{p+1}}\right)_{, i}
\end{aligned}
$$

Shifting $p+1 \rightarrow p$, one finds that the above couplings are exactly given by the second term in (5) in which the RR field strength is given by the second line of (66). This ends our illustration of the consistency between the couplings (5I) and the T-duality. 
We have seen that the consistency with a particular term of the nonlinear T-duality guides us to write the $\mathrm{RR}$ field strength in (5) as $\mathcal{F}=d \mathcal{C}$. The resulting couplings however are consistent with all nonlinear terms of the RR potential. In fact the nonlinear T-duality transformation (35) for the RR potential can be written as [20]

$$
\tilde{\mathcal{C}}_{\mu \cdots \nu \alpha y}^{(n)}=\mathcal{C}_{\mu \cdots \nu \alpha}^{(n-1)} \quad, \quad \tilde{\mathcal{C}}_{\mu \cdots \nu \alpha \beta}^{(n)}=\mathcal{C}_{\mu \cdots \nu \alpha \beta y}^{(n+1)}
$$

The calculations in section 3.1 then show that the couplings (5) with the RR field strength $\mathcal{F}=d \mathcal{C}$ are consistent with the full nonlinear T-duality transformation for the RR field. It would be interesting to confirm the couplings of one RR and two NSNS fields in (5) by the disk level scattering amplitude.

Acknowledgments: I would like to thank Katrin Becker and Rob Myers for useful discussions. This work is supported by Ferdowsi University of Mashhad under grant $\mathrm{p} / 964(1388 / 12 / 4)$.

\section{References}

[1] R. G. Leigh, Mod. Phys. Lett. A 4, 2767 (1989).

[2] C. Bachas, Phys. Lett. B 374, 37 (1996) arXiv:hep-th/9511043.

[3] C. P. Bachas, P. Bain and M. B. Green, JHEP 9905, 011 (1999) arXiv:hep-th/9903210.

[4] M. R. Garousi and R. C. Myers, Nucl. Phys. B 475, 193 (1996) arXiv:hep-th/9603194.

[5] A. Hashimoto and I. R. Klebanov, Phys. Lett. B 381, 437 (1996) arXiv:hep-th/9604065.

[6] M. R. Garousi, JHEP 1002, 002 (2010) [arXiv:0911.0255 [hep-th]].

[7] J. Polchinski, Phys. Rev. Lett. 75, 4724 (1995) [arXiv:hep-th/9510017].

[8] M. R. Douglas, arXiv:hep-th/9512077.

[9] M. B. Green, J. A. Harvey and G. W. Moore, Class. Quant. Grav. 14, 47 (1997) arXiv:hep-th/9605033.

[10] Y. K. Cheung and Z. Yin, Nucl. Phys. B 517, 69 (1998) arXiv:hep-th/9710206.

[11] R. Minasian and G. W. Moore, JHEP 9711, 002 (1997) arXiv:hep-th/9710230.

[12] R. C. Myers, JHEP 9912, 022 (1999) arXiv:hep-th/9910053. 
[13] M. R. Garousi, work in progress.

[14] K. Becker and G. Guo, work in progress.

[15] M. R. Garousi, JHEP 9812, 008 (1998) arXiv:hep-th/9805078.

[16] A. Y. Dymarsky, arXiv:hep-th/0206191.

[17] M. Billo', L. Ferro, M. Frau, F. Fucito, A. Lerda and J. F. Morales, JHEP 0810, 112 (2008) arXiv:0807.1666 [hep-th]].

[18] T. Buscher, Phys. Lett. B 159 (1985) 127; B 194 (1987) 59; B 201 (1988) 466.

[19] P. Meessen and T. Ortin, Nucl. Phys. B 541, 195 (1999) arXiv:hep-th/9806120].

[20] W. Taylor and M. Van Raamsdonk, Nucl. Phys. B 573, 703 (2000) arXiv:hep-th/9910052. 